\title{
Exploitation of a microporous organic polymer as a stationary phase for capillary gas chromatography
}

Cuiming Lu, Shuqin Liu, Jianqiao Xu, Yajuan Ding and Gangfeng Ouyang*

MOE Key Laboratory of Aquatic Product Safety/KLGHEI of Environment and Energy

Chemistry, School of Chemistry and Chemical Engineering, Sun Yat-sen University,

Guangzhou 510275, PR China

Corresponding author. Email: cesoygf@mail.sysu.edu.cn (G. Ouyang); Tel.: +86-020-84110953; Fax: +86-020-84110953. 


\section{Abstract}

Microporous organic polymers (MOPs) have emerged as a new class of functional porous materials with unique characteristics and potential uses in diverse areas. However, the field of MOPs for gas chromatographic (GC) separations has not been well explored. Herein, a MOP namely KAPs-1 was dynamic coated onto a capillary column for the first time. The fabricated column exhibited a nonpolar nature and the column efficiency for $n$-dodecane was up to 7769 plates $\mathrm{m}^{-1}$. The KAPs- 1 coated column showed high GC separation performance for a series of volatile organic compounds (VOCs) including the challenging ethylbenzene and xylene isomers, which could not be resolved at baseline on the commercial 5\% phenyl polysiloxane stationary phase. Moreover, the relative standard deviations for five replicate determinations of the studied analytes were $0.0-0.6 \%, 0.9-3.2 \%, 1.1-5.9 \%, 0.8-3.7 \%$ for retention time, peak area, peak height and peak width, respectively. To investigate the interaction between some analytes and the stationary phase, thermodynamic and kinetic parameters were also evaluated. The results of this study show it is very promising to utilize MOPs as stationary phases for capillary GC.

Keywords: Microporous organic polymers; Gas chromatography; Stationary phase; Volatile organic compounds 


\section{Introduction}

Over the past few decades, great efforts have been devoted to the development of microporous materials featured with average pore sizes less than $2 \mathrm{~nm}$ and the well-known examples include zeolites, activated carbons, silicas, metal organic frameworks (MOFs) and MOPs [1-3]. In particular, MOPs which are composed of light, non-metallic elements (e.g. C, H, O, N, B) [2] have recently become the focus of significant attention owning to their outstanding characteristics and enormous potential in diverse applications.

In general, MOPs could be constructed by linkage of polymerizable monomers as organic building blocks or post-crosslinking of performed polymer chains through kinds of chemical reactions [4,5], exhibiting very broad synthetic diversity in comparison with other microporous solids. Accordingly, the past few years have witnessed the rapid growth of a wide variety of MOPs with different structures and specific properties involving covalent organic frameworks (COFs) [6], conjugated microporous polymers (CMPs) [7], polymers of intrinsic microporosity (PIMs) [8], porous polymer networks (PPNs) [9] and hypercrosslinked polymers (HCPs) [10], etc. However, most of the exploitations of these emerging MOPs have been concentrated on gas separation and storage [11,12], catalysis [13] and sensing [14], fewer data have been presented on their potential serving as stationary phases for GC although there have been two reports on the applications of chiral MOPs (a PPN and a porous organic cage, respectively) in GC separations very recently $[15,16]$. In sharp contrast, MOFs as the inorganic-organic hybrid counterparts of MOPs have been studied 
extensively in the utilizations as new stationary phases for GC [17]. It should be noted that with robust covalent bonds interlinked by organic building blocks instead of weak coordinative interaction [18], MOPs tend to be more stable than MOFs. Therefore, the excellent physicochemical stability coupled with other remarkable properties such as permanent porosity, feasible functionalization and large specific surface area [18-20] renders MOPs to be appealing and possibly superior candidates for separation media in high-resolution GC. The field of MOPs for GC separation remains to be well investigated.

Herein, we report the first example of HCPs based on organic building blocks for fabrication of porous layer open tubular (PLOT) column for GC. The selected novel HCP namely KAPs-1 was prepared by a cost-effective strategy of "knitting" rigid aromatic building blocks with an external cross-linker [21]. This strategy eliminated the need of polymerizable monomers and the use of expensive metal coupling catalysts [4]. In addition, since the sole byproduct was methanol, this strategy was environmentally friendly. KAPs-1 was explored as a potential stationary phase for GC because it possesses the advantages of high surface area, good thermal and chemical stability, predominantly microporosity as well as easy preparation. A series of VOCs were used as targets for separations, including ethylbenzene and xylene isomers of which the separation is significant for both environmental research and chemical industry but challenging because of their close matching boiling points and molecular sizes [22-24]. Furthermore, thermodynamic and kinetic parameters were determined to get insight into the interaction between the stationary phase and some analytes in 
this work.

\section{Experimental}

\subsection{Materials and instruments}

All chemicals are of high purity (analytical grade or higher) and used as supplied without further treatment. $p$-, $m$-, $o$-xylene and toluene were purchased from Alfa Aesar (Beijing, China). Benzene was purchased from Guanghua Chemical Factory Co. Ltd. (Guangdong, China). Sodium hydroxide $(\mathrm{NaOH})$ and hydrochloric acid $(\mathrm{HCl})$ were from Guangzhou Chemical Reagent Company (Guangzhou, China). Methanol was from Sigma-Aldrich (Shanghai, China). The rest chemicals were obtained from Aladdin Chemistry Co. Ltd. (Shanghai, China). Untreated fused silica capillary (30 m long $\times 0.32 \mathrm{~mm}$ i.d.) was purchased from Yongnian Optic Fiber Plant (Hebei, China). A HP-5MS capillary column (30 m long $\times 0.25 \mathrm{~mm}$ i.d. $\times 0.25 \mu \mathrm{m}$ film thickness $)$ was purchased from Agilent Technologies (USA).

Infrared (IR) spectra were recorded on an EQUINOXFourier Transform Infrared spectrometer (FTIR) (Bruker, Germany) in the $400 \sim 4000 \mathrm{~cm}^{-1}$ region. The ${ }^{13} \mathrm{C}$ cross-polarization magic-angle spinning (CP/MAS) NMR spectra were acquired on a Bruker Avance 400 spectrometer at resonance frequencies of $400 \mathrm{MHz}$. Scanning electron microscope (SEM) images were obtained by a Quanta 400F SEM instrument (FEI/Oxford/HKL, Netherlands) at $20 \mathrm{kV}$. Thermogravimetric analyses (TGA) data were collected on a thermal gravimetric analyzer (NETZSCH, Germany) from 30 to $800{ }^{\circ} \mathrm{C}$ at a ramp rate of $10{ }^{\circ} \mathrm{C} \min ^{-1}$ under $\mathrm{N}_{2}$. Transmission electron microscope (TEM) micrographs were acquired on a Tecnai G2 Spirit microscope (FEI, 
Netherlands) operating at a $120 \mathrm{kV}$ accelerating voltage. $\mathrm{N}_{2}$ adsorption-desorption isotherms were measured at $77.3 \mathrm{~K}$ on Micromeritics ASAP 2020 V3.04 H surface area and porosity analyzer based on the Brunauer, Emmet, and Teller (BET) method. GC measurements were performed on an Agilent 6850 GC system equipped with a flame ionization detector (FID). Nitrogen (99.999\%) and Hydrogen (99.999\%) were used as the carrier gas and the fuel gas, respectively. The inlet temperature was set at $250{ }^{\circ} \mathrm{C}$ and the detector temperature was $280^{\circ} \mathrm{C}$.

\subsection{Synthesis of KAPs-1}

KAPs-1 was obtained by a simple one-step Friedel-Crafts reaction according to Li et al [21]. Typically, benzene $(0.02 \mathrm{~mol})$ as the monomers and formaldehyde dimethyl acetal $(0.06 \mathrm{~mol})$ as cross linkers were dissolved in 1,2-dichloroethane (20 $\mathrm{mL}$ ), and then the catalyst (anhydrous $\mathrm{FeCl}_{3}, 0.06 \mathrm{~mol}$ ) was introduced into the mixture solution and mixed well at room temperature. The reaction system was stirred at $45^{\circ} \mathrm{C}$ for $5 \mathrm{~h}$ to achieve initial networks and subsequently heated at $80{ }^{\circ} \mathrm{C}$ for $19 \mathrm{~h}$ to complete the condensation reaction. After filtrated and washed with methanol thoroughly, the product was collected by Soxhlet extraction in methanol for $24 \mathrm{~h}$. Finally, the brown powders were obtained by drying in vacuo at $60^{\circ} \mathrm{C}$ for $24 \mathrm{~h}$.

\subsection{Preparation of KAPs-1 coated capillary column}

The pretreatment of the capillary column prior to coating was as follows: untreated fused silica capillary was washed sequentially with $1 \mathrm{M} \mathrm{NaOH}$ for $2 \mathrm{~h}$, ultrapure water for $0.5 \mathrm{~h}, 0.1 \mathrm{M} \mathrm{HCl}$ for $2 \mathrm{~h}$ and again with ultrapure water until the washings were neutral. The capillary was then purged with nitrogen at $150{ }^{\circ} \mathrm{C}$ 
overnight.

The KAPs- 1 coated capillary column was prepared by a dynamic coating method [25]: $2.5 \mathrm{mg}$ KAPs-1 was dispersed in $2.0 \mathrm{~mL}$ toluene and sonicated for $10 \mathrm{~min}$. After set aside for $15 \mathrm{~min}$, the supernatant of the suspension was collected, filled into the capillary as a plug and then pushed through the capillary with nitrogen at a rate of $30 \mathrm{~cm} \mathrm{~min}^{-1}$. A $1 \mathrm{~m}$ long buffer tube $(0.32 \mathrm{~mm}$ i.d.) as a restrictor was attached to the end of the capillary to avoid the acceleration of the solution plug near the end of the capillary. The resulting wet coating layer on the inner wall of the capillary was dried under nitrogen overnight. Finally, the KAPs-1 coated capillary column was conditioned by increasing its temperature at a rate of $1{ }^{\circ} \mathrm{C} \mathrm{min}-1$ from 30 to $270{ }^{\circ} \mathrm{C}$ (held for $30 \mathrm{~min}$ ) and then decreasing to $30{ }^{\circ} \mathrm{C}$ at the same rate. The procedure was repeated for three times.

\subsection{Thermodynamic analysis}

Thermodynamic parameters involving enthalpy change $\Delta H$, entropy change $\Delta S$ and Gibbs free energy change $\Delta G$, which could elucidate the energy effect on the interaction between targets and the studied stationary phase, were calculated on the basis of the van't Hoff model [26] (Eqs. (1) and (2)).

$$
\begin{gathered}
\ln k^{\prime}=\frac{-\Delta H}{R T}+\frac{\Delta S}{R}+\ln \phi \\
\Delta G=\Delta H-T \Delta S
\end{gathered}
$$

where $k^{\prime}$ represents the retention factor, $R$ the gas constant, $T$ the absolute temperature and $\phi$ the phase patio, which is defined as the volume of the stationary phase divided by the volume of the mobile phase. In this study, the calculated value of 
$\phi$ was $9.44 \times 10^{-3}$ according to the thickness of the coating (approximately $1.5 \mu \mathrm{m}$ ).

The enthalpy-entropy compensation was applied to chromatographic system to evaluate the retention mechanism [27], which could be expressed by the following formula:

$$
\Delta H=\beta \Delta S+\Delta G_{\beta}
$$

Here $\Delta G_{\beta}$ is the Gibbs free energy of a physicochemical interaction at the compensation temperature $\beta$.

Combining Eqs. (1) and (3), Eqs. (4) could be obtained:

$$
\ln k^{\prime}=\frac{-\Delta H}{R}\left(\frac{1}{T}-\frac{1}{\beta}\right)-\frac{\Delta G_{\beta}}{R \beta}+\ln \phi
$$

Therefore, a plot of $\ln k^{\prime}$ against $\Delta H$ could be used to assess the enthalpy-entropy compensation.

\subsection{Kinetic evaluation}

The peak broadening in open tubular capillaries is generally described by the Golay equation as follows [28]:

$$
\begin{gathered}
H=\frac{B}{u}+C \times u \\
H=\frac{B}{u}+\left(C_{M}+C_{S}\right) \times u
\end{gathered}
$$

Here $H$ is for height equivalent to a theoretical plate (HETP), $u$ is the average linear velocity of the mobile phase, $B$ stands for the coefficient of longitudinal diffusion and $C$, the coefficient of mass transfer resistance. By expanding $B$ and $C$ fully, a more thorough version of Equation (5) was presented:

$$
H=\frac{2 D_{M}}{u}+\left[\frac{1+6 k^{\prime}+11 k^{\prime 2}}{96\left(1+k^{\prime}\right)^{2}} \times \frac{d_{c}^{2}}{D_{M}}+\frac{2 k^{\prime}}{3\left(1+k^{\prime}\right)^{2}} \times \frac{d_{f}^{2}}{D_{S}}\right] \times u
$$


In which $k^{\prime}$ is the retention factor; $D_{M}$ and $D_{S}$ are the diffusion coefficients of the analytes in the mobile phase and stationary phase, respectively; $C_{M}$ and $C_{S}$ denote the mass transfer resistance coefficient in the mobile phase and stationary phase, respectively; $d_{c}$ is the inner diameter of the capillary column; $d_{f}$ is the thickness of the stationary phase.

\section{Results and discussion}

\subsection{Characterization of the synthesized KAPs-1 and the KAPs-1 coated column}

Several characterization techniques were routinely employed to testify the successful synthesis of KAPs-1. The FTIR spectrum (Fig. 1a) displayed the characteristic adsorption peaks in the range of $1650-1450 \mathrm{~cm}^{-1}$ derived from the skeleton vibrations of the benzene rings. In the ${ }^{13} \mathrm{C}$ CP/MAS NMR spectrum (Fig. 1b), the peaks at 141.60 and $134.67 \mathrm{ppm}$ were assignable to the aromatic carbon atoms and the signals near $40 \mathrm{ppm}$ were ascribed to the carbon in methylene of the KAPs- 1 . The porosity of KAPs-1 was illustrated by the $\mathrm{N}_{2}$ adsorption-desorption isotherms at 77.3 $\mathrm{K}$ (Fig. 1c) and the BET surface area was evaluated to be $1434 \mathrm{~m}^{2} \mathrm{~g}^{-1}$. As shown in Fig. 1d, KAPs- 1 was thermally stable up to $300{ }^{\circ} \mathrm{C}$, which suggested it was suitable for GC application.

TEM image (Fig. 2a) demonstrated that the synthesized KAPs-1 was amorphous in essence with particle size less than $100 \mathrm{~nm}$. Fig. 2b and 2c presented SEM images of the fabricated capillary column in the cross-section view and clearly, an approximately $1.5 \mu \mathrm{m}$ uniform coating was deposited well on the inner wall. The number of theoretical plates of the KAPs- 1 coated column measured by $n$-dodecane at 
$150{ }^{\circ} \mathrm{C}$ was up to 7769 plates $\mathrm{m}^{-1}$, implying the considerably high column efficiency. Polarity of a stationary phase, which plays a key role in the selectivity of a GC column, is usually described by McReynolds constants [29,30]. In this work, McReynolds constants were measured at $120{ }^{\circ} \mathrm{C}$ by using benzene, 1-butanol, 2-pentanone, 1-nitropropane and pyridine as probe compounds. A non-polar, commercial capillary column (HP-5MS) containing 5\% phenyl polysiloxane stationary phase was employed for comparison. As shown in Table S1 (Electronic Supplementary Information), the average value of the five McReynolds constants of the novel stationary phase was smaller than that of the commercial stationary phase. Consequently, the KAPs-1 coated column could also be identified as non-polar.

\subsection{Separation performance of the KAPs-1 coated column}

To examine the separation performance of the KAPs- 1 coated column, several kinds of VOCs of which the separation and analysis is of huge practical interest were chosen as test targets, including linear alkanes $\left(\mathrm{C}_{5}-\mathrm{C}_{12}\right)$, chlorobenzenes, nitrobenzenes and BTEXs (the mixture of benzene, toluene, ethylbenzene and xylene isomers). Fig. 3 illustrated the corresponding GC chromatograms for the separation of the four groups of VOCs. Although covering a wide range of boiling points (from $36{ }^{\circ} \mathrm{C}$ to $242{ }^{\circ} \mathrm{C}$ ), all of the targets achieved baseline separation with nice peak shapes within relatively short time. By temperature-programmed control, the elution sequences of linear alkane mixtures, chlorobenzenes and nitrobenzenes on the KAPs-1 coated column were in good accordance with their incremental order of boiling points, respectively, which was also observed on the non-polar HP-5MS. It 
could be inferred that van der Waals forces mainly influenced the retention of these analytes on the KAPs-1 coated column.

Typically, ethylbenzene and xylene isomers (also known as C8 alkylaromatics) are difficult to be resolved at baseline which is mainly ascribed to the extremely high resemblance of their physical properties (Electronic Supplementary Information, Table S2). Commercial capillaries coated with polyethylene glycol with strong polarity and a PLOT column prepared by MIL-101 (a kind of MOFs) with weak polarity had been reported to separate the mixture very well via GC [23,25]. The elution sequence of ethylbenzene and xylene isomers on these two stationary phases followed the order of their boiling points and polarity, respectively. However, in the case of KAPs-1 stationary phase, the non-polar nature offered good selectivity for the separation of BTEXs without the requirement for temperature-programming (Fig. 3d). Moreover, the order of elution of BTEXs did not exactly correspond to that of their boiling points where $m$-xylene with higher boiling point exceptionally eluted faster than $p$-xylene. It is likely that a different retention mechanism occurred on the KAPs-1 coating, thus, the interaction between BTEXs and the KAPs-1 coating was elucidated hereinafter in terms of thermodynamics and kinetics.

\subsection{Thermodynamic analysis}

The dependence of the retention factors for BTEXs on the temperature according to the van't Hoff equation was plotted in Fig. 4a, from which the enthalpy change $(\Delta H)$ and the entropy change $(\Delta S)$ for transfer of the analyte from the mobile to the stationary phase were calculated (Table 1). Additionally, the enthalpy-entropy 
compensation for the separation of BTEXs was examined (Fig. 4b). The excellent linearity of the compensation chart as well as the van't Hoff plots revealed that the retentions on the stationary phase associated with temperature for all of the six compounds were governed by an essentially identical interaction mechanism and there was no change over the test temperature range [26].

As reported in other stationary phases [32], the targets with the more negative values of Gibbs free energy change $(\Delta G)$ were retained more strongly in the column due to their larger inclination to transfer from the mobile to the stationary phase (Table 1). It's worth noting that there were only subtle distinctions for different C8 alkylaromatics in $\Delta H$ and $\Delta S$, respectively, indicative of their comparable interaction with the micropores in the KAPs-1 coating (Table 1). The micropore system of the MOP appeared to be advantageous to generate the differences in adsorption between these isomers. As for $p$-xylene and $m$-xylene which have close matching boiling points, pore-filling effect may be dominant among other factors for their retention [23]. Compared with $m$-xylene, the configuration of $p$-xylene facilitated its more efficient packing in the confined available space in the micropores of which the pore walls were filled with benzene rings. The MOP thus showed preferential retention of $p$-xylene over $m$-xylene. The larger negative values of $\Delta H$ and $\Delta S$ of $p$-xylene also confirmed that it interacted with the pore walls more strongly, was adsorbed more tightly and lost more freedom. Hence, $p$-xylene eluted after $m$-xylene, independent of their boiling points.

\subsection{Kinetic evaluation}


In general, the resolution of a pair analytes is controlled by both selectivity and peak width. Selectivity is believed to be related to thermodynamics of the retention while peak width is usually affected by kinetics. Height equivalent to a theoretical plate (HETP) is used as a measure of the amount of peak broadening that occurs when an analyte pass through the column [28]. In this study, the variations of HETP with the average linear velocities of the mobile phase for BTEXs were fitted on the basis of the Golay equation for peak broadening in open tubular capillaries (Fig. 5) and the relevant parameters, namely longitudinal diffusion coefficient $B$ and mass transfer resistance coefficient $C$, were available.

Longitudinal diffusion originates from the concentration gradient of an analyte when passing through the column. Mass transfer resistance is related to the time an analyte molecule needs to transfer between the stationary phase and the mobile phase [33]. $H_{\min }$ is the minimal value of HETP for a specific analyte under the optimal velocity of the mobile phase. The larger $H_{\min }$ results in wider chromatographic peaks. As shown in Fig. 6, values of $B$ and $C$ shared almost the same trend for the six analytes. The analyte with larger $B$ and $C$ had a bigger value of $H_{\min }$, which implied both longitudinal diffusion and mass transfer played important roles in the peak broadening. In contrast with $o$-xylene, the other three isomers (ethylbenzene, $p$-xylene and $m$-xylene) had much lower longitudinal diffusion and resistance to mass transfer, which help to avoid peaks overlapped with one another and receive baseline separation. The diffusion coefficients of the analytes in the mobile phase $\left(D_{M}\right)$ and stationary phase $\left(D_{S}\right)$ along with $C_{M}$ and $C_{S}$, the terms for the mass transfer resistance 
in the mobile and stationary phase, respectively, could be obtained by expanding $B$ and $C$ [34], to gain further insight into the kinetics of the separation (Electronic Supplementary Information, Fig. S1).

\subsection{Column repeatability and stability}

The KAPs-1 coated column showed good repeatability in separation performance. The relative standard deviations for five replicate determinations of the targets were $0.0-0.6 \%, 0.9-3.2 \%, 1.1-5.9 \%, 0.8-3.7 \%$ for retention time, peak area, peak height and peak width, respectively (Electronic Supplementary Information, Table S3). Excellent stability was also observed on the KAPs-1 coated column. As shown in Fig. 7, the chromatograms of chlorobenzenes and nitrobenzenes before and after repeated use were reproducible. Although the KAPs-1 coated column performed more than 700 chromatographic separations and was set aside for five months at the ambient conditions, there was no discernible loss of separation power on the KAPs-1 stationary phase.

\section{Conclusions}

In summary, the successfully fabricated KAPs-1 coated PLOT column exhibited great promise for GC separation of the VOCs studied, especially the challenging ethylbenzene and xylene isomers. By virtue of their intriguing features, development of emerging MOPs as novel stationary phases for GC would be a promising field and is bound to broaden the window of applications of MOPs.

\section{Acknowledgements}

We acknowledge the financial support from the projects of NNSFC (21225731, 
21477166) and the NSF of Guangdong Province (S2013030013474) for this work.

\section{References:}

[1] R. Dawson, A.I. Cooper, D.J. Adams, Nanoporous organic polymer networks, Prog. Polym. Sci. 37 (2012) 530-563.

[2] J.X. Jiang, A.I. Cooper, Microporous organic polymers: Design, synthesis, and function, Top. Curr. Chem. 293 (2010) 1-33.

[3] S. Kim, Y.M. Lee, Rigid and microporous polymers for gas separation membranes, Prog. Polym. Sci. 43 (2015) 1-32.

[4] S.J. Xu, Y.L. Luo, B.E. Tan, Recent development of hypercrosslinked microporous organic polymers, Macromol. Rapid Commun. 34 (2013) 471-484.

[5] D.C. Wu, F. Xu, B. Sun, R.W. Fu, H.K. He, K. Matyjaszewski, Design and preparation of porous polymers, Chem. Rev. 112 (2012) 3959-4015.

[6] H.M. El-Kaderi, J.R. Hunt, J.L. Mendoza-Cortés, A.P. Côté, R.E. Taylor, M. O'Keeffe, O.M. Yaghi, Designed synthesis of 3D covalent organic frameworks, Science 316 (2007) 268-272.

[7] N.B. McKeown, S. Makhseed, P.M. Budd, Phthalocyanine-based nanoporous network polymers, Chem. Commun. 23 (2002) 2780-2781.

[8] J.X. Jiang, F. Su, A. Trewin, C.D. Wood, N.L. Campbell, H. Niu, C. Dickinson, A.Y. Ganin, M.J. Rosseinsky, Y.Z. Khimyak, A.I. Cooper, 
Conjugated microporous poly(aryleneethynylene) networks, Angew. Chem. Int. Ed. 46 (2007) 8574-8578.

[9] W.G. Lu, D.Q. Yuan, J. Sculley, D. Zhao, R. Krishna, H.C. Zhou, Sulfonate-grafted porous polymer networks for preferential $\mathrm{CO}_{2}$ adsorption at low pressure, J. Am. Chem. Soc. 133 (2011) 18126-18129.

[10]C.D. Wood, B.E. Tan, A. Trewin, H.J. Niu, D. Bradshaw, M.J. Rosseinsky, Y.Z. Khimyak, N.L. Campbell, R. Kirk, E. Stöckel, A.I. Cooper, Hydrogen storage in microporous hypercrosslinked organic polymer networks, Chem. Mater. 19 (2007) 2034-2038.

[11]M. Carta, R. Malpass-Evans, M. Croad, Y. Rogan, J.C. Jansen, P. Bernardo, F. Bazzarelli, N.B. McKeown, An efficient polymer molecular sieve for membrane gas separations, Science 339 (2013) 303-307.

[12]A. Nagai, Z.Q. Guo, X. Feng, S.B. Jin, X. Chen, X.S. Ding, D.L. Jiang, Pore surface engineering in covalent organic frameworks, Nat. Commun. 2 (2011) 536.

[13]R.K. Totten, Y.S. Kim, M.H. Weston, O.K. Farha, J.T. Hupp, S.T. Nguyen, Enhanced catalytic activity through the tuning of micropore environment and supercritical $\mathrm{CO}_{2}$ processing: $\mathrm{Al}($ Porphyrin)-based porous organic polymers for the degradation of a nerve agent simulant, J. Am. Chem. Soc. 135 (2013) 11720-11723.

[14]X.M. Liu, Y.H. Xu, D.L. Jiang, Conjugated microporous polymers as molecular sensing devices: Microporous architecture enables rapid response 
and enhances sensitivity in fluorescence-on and fluorescence-off sensing, J. Am. Chem. Soc. 134 (2012) 8738-8741.

[15]J.Q. Dong, Y. Liu, Y. Cui, Chiral porous organic frameworks for asymmetric heterogeneous catalysis and gas chromatographic separation, Chem. Commun. 50 (2014) 14949-14952.

[16]A. Kewley, A. Stephenson, L.J. Chen, M.E. Briggs, T. Hasell, A.I. Cooper, Porous organic cages for gas chromatography separations, Chem. Mater. 27 (2015) 3207-3210.

[17]Y.B. Yu, Y.Q. Ren, W. Shen, H.M. Deng, Z.Q. Cao, Applications of metal-organic frameworks as stationary phases in chromatography, Trends Anal. Chem. 50 (2013) 33-41.

[18]Z. Chang, D. S.Zhang, Q. Chen and X. H. Bu, Microporous organic polymers for gas storage and separation applications, Phys. Chem. Chem. Phys. 15 (2013) 5430-5442.

[19]D.Q. Yuan, W.G. Lu, D. Zhao, H.C. Zhou, Highly stable porous polymer networks with exceptionally high gas-uptake capacities, Adv. Mater. 23 (2011) 3723-3725.

[20]Z.Z. Yang, Y.F. Zhao, H.Y. Zhang, B. Yu, Z.S. Ma, G.P. Ji, Z.M. Liu, Fluorinated microporous organic polymers: Design and applications in $\mathrm{CO}_{2}$ adsorption and conversion, Chem. Commun. 50 (2014) 13910-13913.

[21]B.Y. Li, R.N. Gong, W. Wang, X. Huang, W. Zhang, H.M. Li, C.X. Hu, B.E. Tan, A new strategy to microporous polymers: Knitting rigid aromatic 
building blocks by external cross-linker, Macromolecules 44 (2011) $2410-2414$.

[22]M.P.M. Nicolau, P.S. Bárcia, J.M. Gallegos, J.A.C. Silva, A.E. Rodrigues, B.L. Chen, Single- and multicomponent vapor-phase adsorption of xylene isomers and ethylbenzene in a microporous metal-organic framework, J. Phys. Chem. C 113 (2009) 13173-13179.

[23]V. Finsy, H. Verelst, L. Alaerts, D. De Vos, P.A. Jacobs, G.V. Baron, J.F.M. Denayer, Pore-filling-dependent selectivity effects in the vapor-phase separation of xylene isomers on the metal-organic framework MIL-47, J. Am. Chem. Soc. 130 (2008) 7110-7118.

[24]N. Yassaa, E. Brancaleoni, M. Frattoni, P. Ciccioli, Isomeric analysis of BTEXs in the atmosphere using $\beta$-cyclodextrin capillary chromatography coupled with thermal desorption and mass spectrometry, Chemosphere 63 (2006) 502-508.

[25]Z.Y. Gu, X.P. Yan, Metal-organic framework MIL-101 for high-resolution gas chromatographic separation of xylene isomers and ethylbenzene, Angew. Chem. Int. Ed. 49 (2010) 1477-1480.

[26]E. Peyrin, Y.C. Guillaume, A. Villet, A. Ravel, C. Grosset, J. Alary, 18-crown-6-aminophenol isomer complexes studied by RPLC, Chromatographia 52 (2000) 584-588. 
[27]L.A. Cole, J.G. Dorsey, Temperature dependence of retention in reversed-phase liquid chromatography. 1. stationary-phase considerations, Anal. Chem. 64 (1992) 1317-1323.

[28]V.R. Reid, R.E. Synovec, High-speed gas chromatography: The importance of instrumentation optimization and the elimination of extra-column band broadening, Talanta 76 (2008) 703-717.

[29]Z.Y. Gu, J.Q. Jiang, X.P. Yan, Fabrication of isoreticular metal-organic framework coated capillary columns for high-resolution gas chromatographic separation of persistent organic pollutants, Anal. Chem. 83 (2011) 5093-5100.

[30]A. Berthod, E.Y. Zhou, K. Le, D.W. Armstrong, Determination and use of Rohrschneider-McReynolds constants for chiral stationary phases used in capillary gas chromatography, Anal. Chem. 67 (1995) 849-857.

[31]J. Fan, Z.Z. Wang, Q. Li, M.L. Qi, S.J. Shao, R.N. Fu, Calix[4]pyrroles: Highly selective stationary phases for gas chromatographic separations, J. Chromatogr. A 1362 (2014) 231-240.

[32]N. Chang, X.P. Yan, Exploring reverse shape selectivity and molecular sieving effect of metal-organic framework UIO-66 coated capillary column for gas chromatographic separation, J. Chromatogr. A 1257 (2012) 116-124.

[33]A.S. Münch, J. Seidel, A. Obst, E. Weber, F.O.R.L. Mertens, High-separation performance of chromatographic capillaries coated with MOF-5 by the controlled SBU approach, Chem. Eur. J. 17 (2011) 10958-10964. 


\section{Figure captions}

Fig. 1. Characterization of synthesized KAPs-1: (a) FTIR spectrum; (b) The ${ }^{13} \mathrm{C}$ CP/MS NMR spectrum (signals with * are spinning sidebands); (c) $\mathrm{N}_{2}$ adsorption-desorption isotherms at $77.3 \mathrm{~K}$ and (d) TGA curve.

Fig. 2. (a) TEM image of synthesized KAPs-1 and SEM images of the cross section of KAPs-1 coated capillary column at (b) $600 \times$ and (c) $8000 \times$.

Fig. 3. GC chromatograms on the KAPs- 1 coated capillary column $(30 \mathrm{~m}$ long $\times 0.32$ mm i.d.) for separation of (a) n-alkanes: $80{ }^{\circ} \mathrm{C}$ for $1.5 \mathrm{~min}$ and then $30{ }^{\circ} \mathrm{C} \min ^{-1}$ to $250{ }^{\circ} \mathrm{C}$ (held for $3 \mathrm{~min}$ ) under a $\mathrm{N}_{2}$ flow rate of $1.5 \mathrm{~mL} \mathrm{~min}^{-1}$; (b) chlorobenzenes: $180{ }^{\circ} \mathrm{C}$ for $2 \mathrm{~min}$ and then $30{ }^{\circ} \mathrm{C} \min ^{-1}$ to $250{ }^{\circ} \mathrm{C}$ (held for $2.5 \mathrm{~min}$ ) under a $\mathrm{N}_{2}$ flow rate of $0.8 \mathrm{~mL} \mathrm{~min}^{-1}$; (c) nitrobenzenes: $210{ }^{\circ} \mathrm{C}$ for $2 \mathrm{~min}$ and then $30{ }^{\circ} \mathrm{C} \min ^{-1}$ to $250{ }^{\circ} \mathrm{C}$ (held for $2.5 \mathrm{~min}$ ) under a $\mathrm{N}_{2}$ flow rate of $0.8 \mathrm{~mL} \mathrm{~min}^{-1}$; (d) BTEXs: at a $\mathrm{N}_{2}$ flow rate of $0.8 \mathrm{~mL} \mathrm{~min}^{-1}$ under $170{ }^{\circ} \mathrm{C}$.

Fig. 4. (a) Van't Hoff plots and (b) enthalpy-entropy compensation chart for BTEXs on the KAPs-1 coated capillary column.

Fig. 5. Golay plots showing the dependence of HETP on the average linear velocity of the mobile phase for BTEXs at constant temperature of $170{ }^{\circ} \mathrm{C}$.

Fig. 6. (a) Minimal plate height (black), longitudinal diffusion coefficient $B$ (red) and mass transfer resistance coefficient $C$ (blue) of BTEXs; (b) diffusion coefficients in the mobile phase $D_{M}$ (red) and stationary phase $D_{S}$ (blue) of BTEXs and (c) mass transfer resistance coefficients in the mobile phase $C_{M}$ (red) and stationary phase $C_{S}$ (blue) of BTEXs. 
Fig. 7. Reproducible chromatograms on the KAPs-1 coated capillary column (30 m long $\times 0.32 \mathrm{~mm}$ i.d.) for separation of (a) chlorobenzenes: $180{ }^{\circ} \mathrm{C}$ for $2 \mathrm{~min}$ and then $30{ }^{\circ} \mathrm{C} \min ^{-1}$ to $250{ }^{\circ} \mathrm{C}$ (held for $2.5 \mathrm{~min}$ ) under a $\mathrm{N}_{2}$ flow rate of $0.8 \mathrm{~mL} \mathrm{~min}^{-1}$ and (b) nitrobenzenes: $210{ }^{\circ} \mathrm{C}$ for $2 \mathrm{~min}$ and then $30{ }^{\circ} \mathrm{C} \min ^{-1}$ to $250{ }^{\circ} \mathrm{C}$ (held for $2.5 \mathrm{~min}$ )

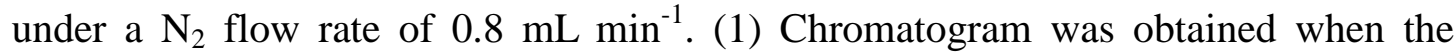
targets were first separated on the KAPs-1 coated column. $(2,3)$ Chromatograms were obtained after more than 700 injections (in the range of $50-250{ }^{\circ} \mathrm{C}$ ) and set aside for five months at the ambient conditions. 
Fig. 1. Characterization of synthesized KAPs-1: (a) FTIR spectrum; (b) The ${ }^{13} \mathrm{C}$ CP/MS NMR spectrum (signals with $*$ are spinning sidebands); (c) $\mathrm{N}_{2}$ adsorption-desorption isotherms at $77.3 \mathrm{~K}$ and (d) TGA curve.

(a)
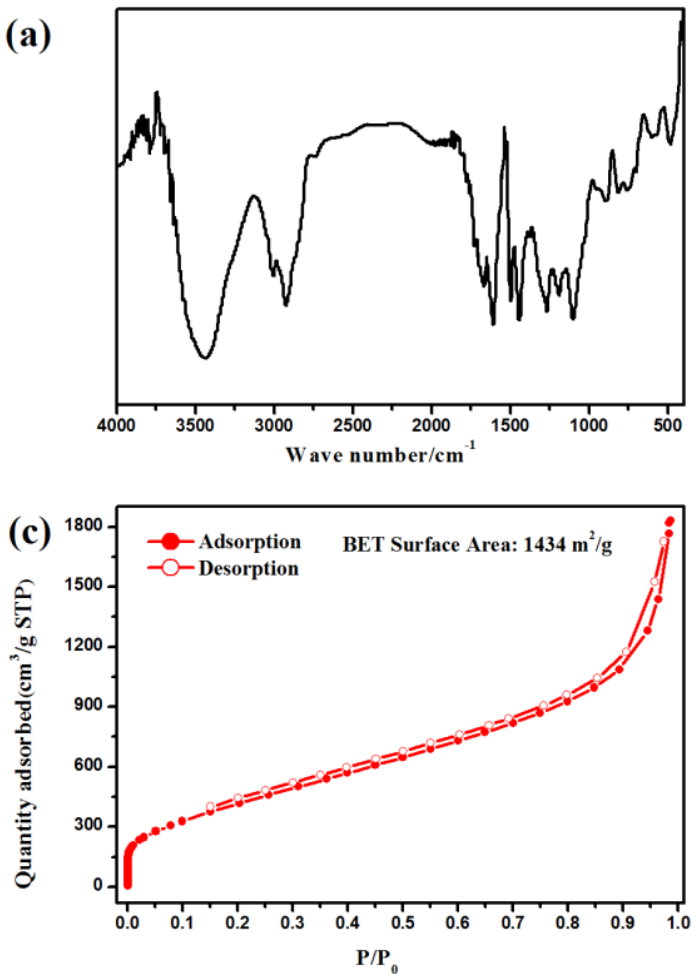

(b)

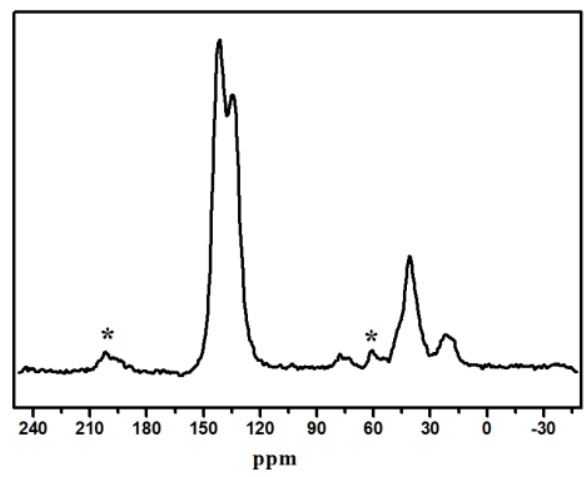

(d)

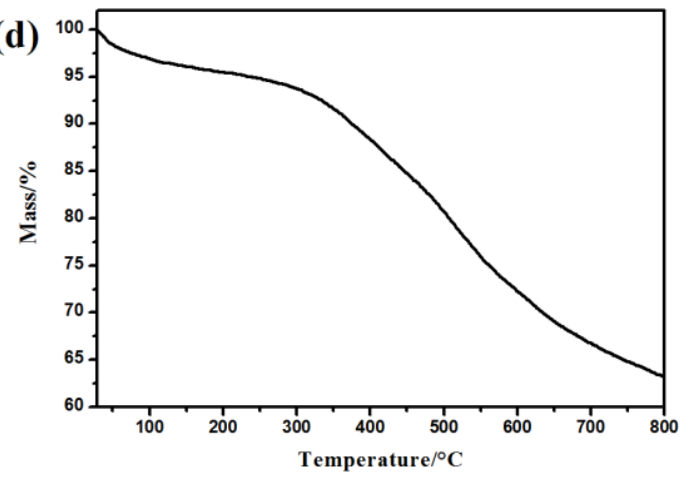


Fig. 2. (a) TEM image of synthesized KAPs-1 and SEM images of the cross section of KAPs- 1 coated capillary column at (b) $600 \times$ and (c) $8000 \times$.

(a)

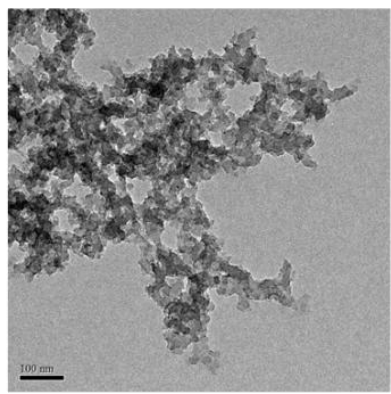

(b)

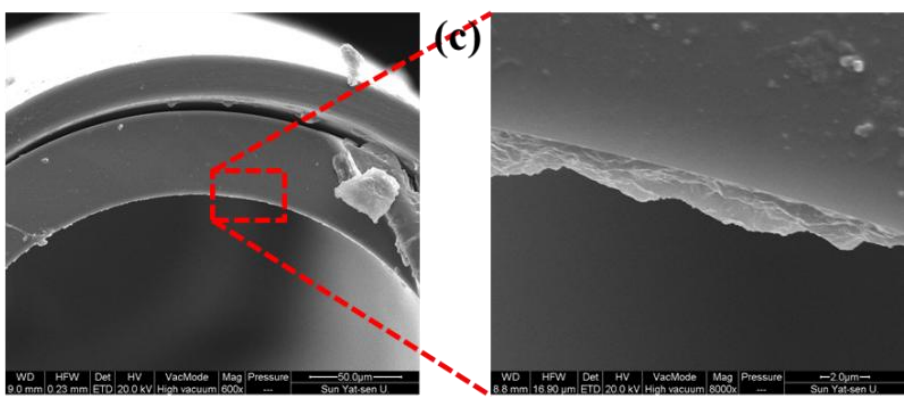


Fig. 3. GC chromatograms on the KAPs- 1 coated capillary column $(30 \mathrm{~m}$ long $\times 0.32$ mm i.d.) for separation of (a) n-alkanes: $80{ }^{\circ} \mathrm{C}$ for $1.5 \mathrm{~min}$ and then $30{ }^{\circ} \mathrm{C} \min ^{-1}$ to $250{ }^{\circ} \mathrm{C}$ (held for $3 \mathrm{~min}$ ) under a $\mathrm{N}_{2}$ flow rate of $1.5 \mathrm{~mL} \mathrm{~min}^{-1}$; (b) chlorobenzenes: $180{ }^{\circ} \mathrm{C}$ for $2 \mathrm{~min}$ and then $30{ }^{\circ} \mathrm{C} \min ^{-1}$ to $250{ }^{\circ} \mathrm{C}$ (held for $2.5 \mathrm{~min}$ ) under a $\mathrm{N}_{2}$ flow rate of $0.8 \mathrm{~mL} \mathrm{~min}^{-1}$; (c) nitrobenzenes: $210{ }^{\circ} \mathrm{C}$ for $2 \mathrm{~min}$ and then $30{ }^{\circ} \mathrm{C} \min ^{-1}$ to $250{ }^{\circ} \mathrm{C}$ (held for $2.5 \mathrm{~min}$ ) under a $\mathrm{N}_{2}$ flow rate of $0.8 \mathrm{~mL} \mathrm{~min}^{-1}$; (d) BTEXs: at a $\mathrm{N}_{2}$ flow rate of $0.8 \mathrm{~mL} \mathrm{~min}^{-1}$ under $170{ }^{\circ} \mathrm{C}$.

(a)

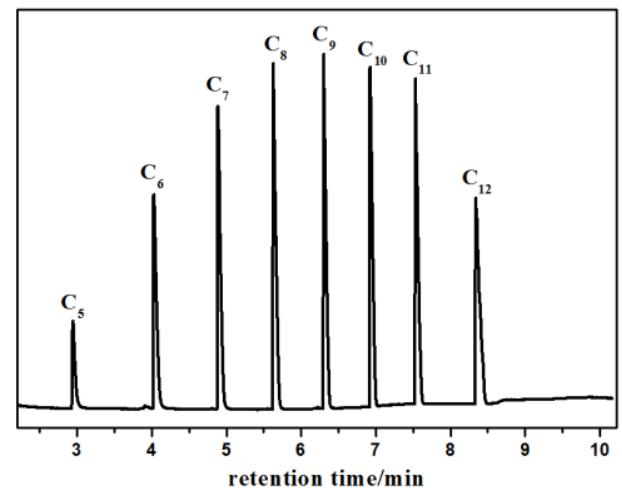

(c)

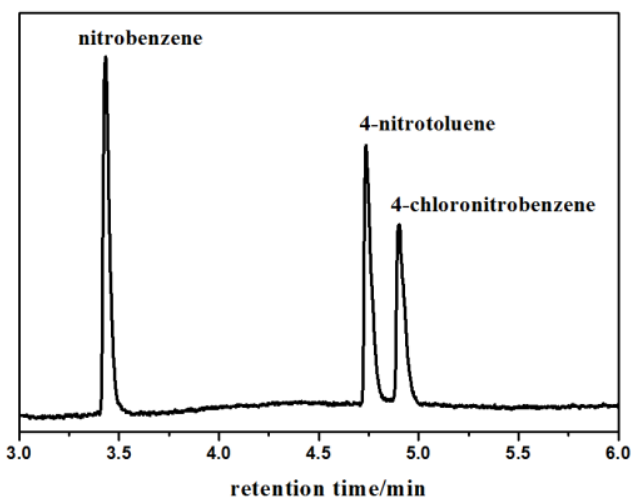

(b)

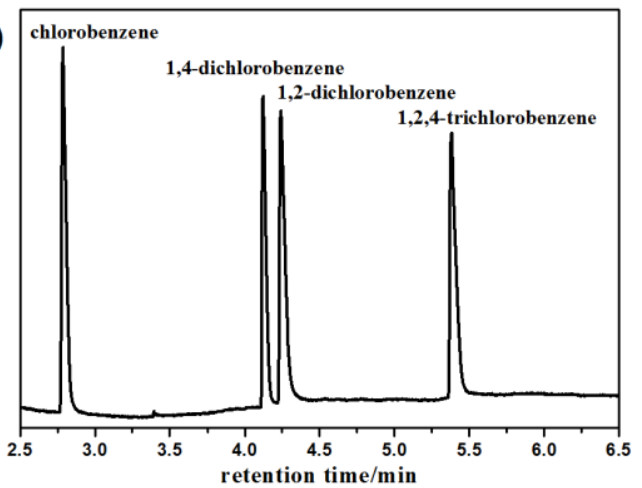

(d)

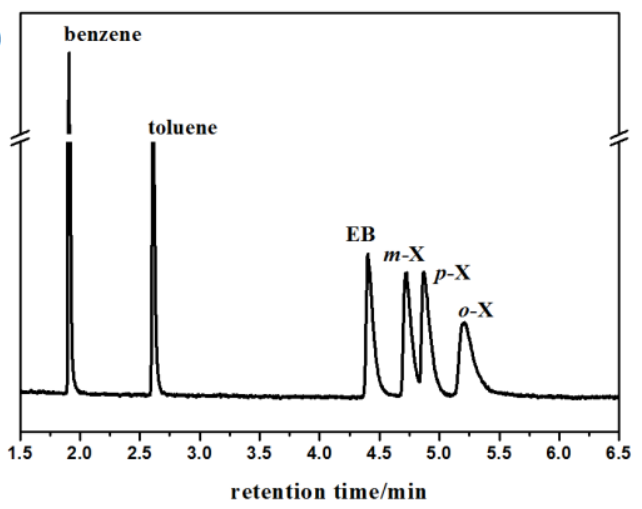


Fig. 4. (a) Van't Hoff plots and (b) enthalpy-entropy compensation chart for BTEXs on the KAPs-1 coated capillary column.

(a)

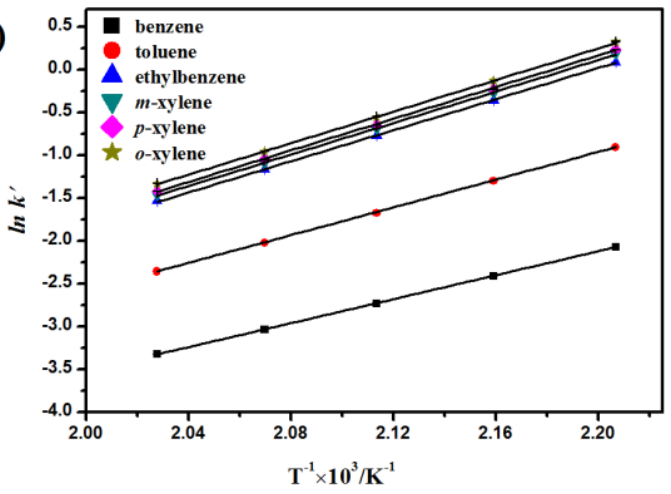

(b)

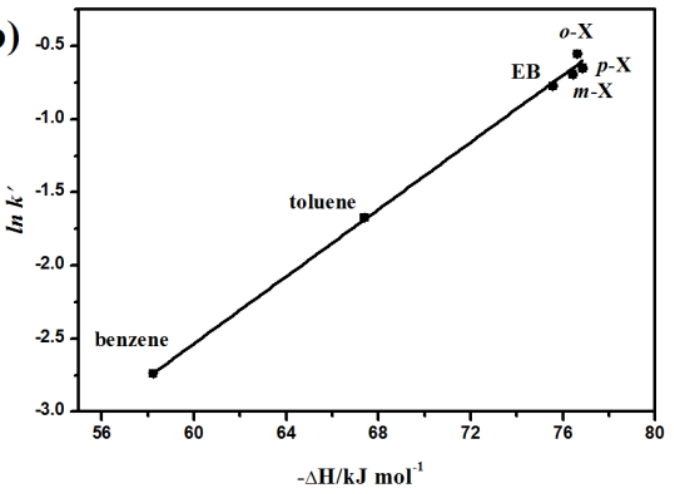


Fig. 5. Golay plots showing the dependence of HETP on the average linear velocity of the mobile phase for BTEXs at constant temperature of $170{ }^{\circ} \mathrm{C}$.

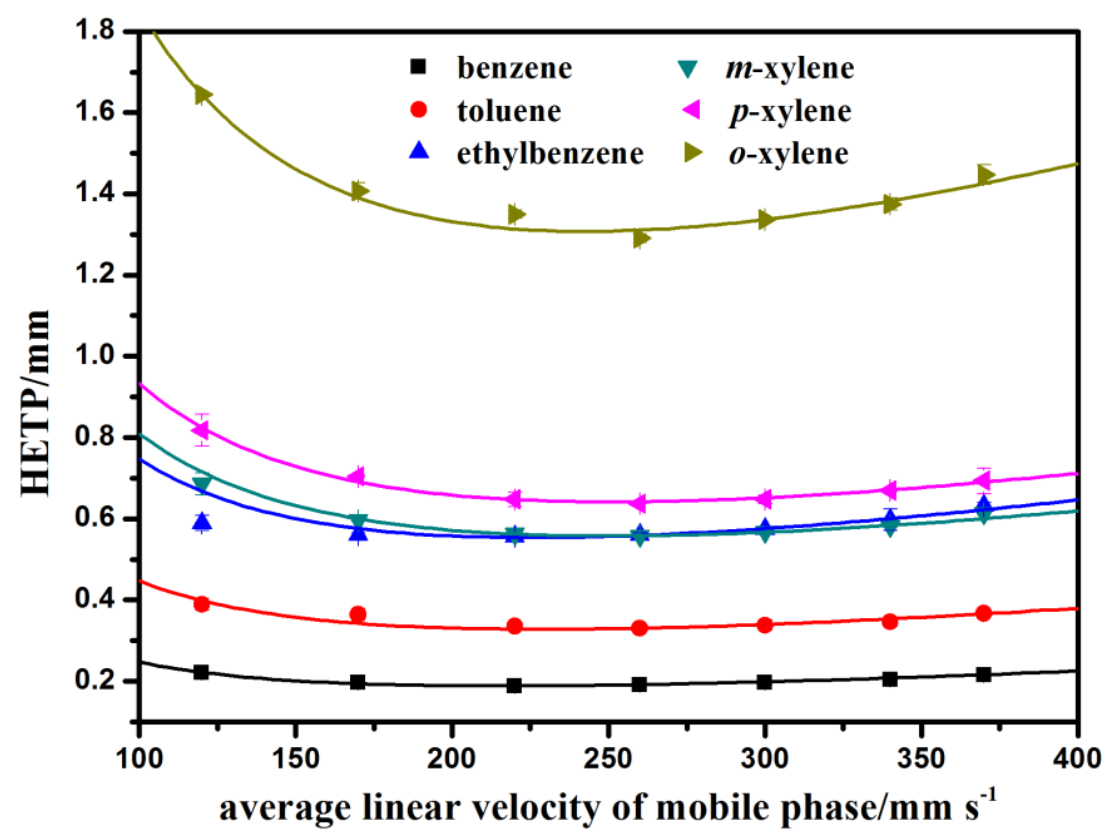


Fig. 6. Minimal plate height (black), longitudinal diffusion coefficient $B$ (red) and mass transfer resistance coefficient $C$ (blue) of BTEXs.

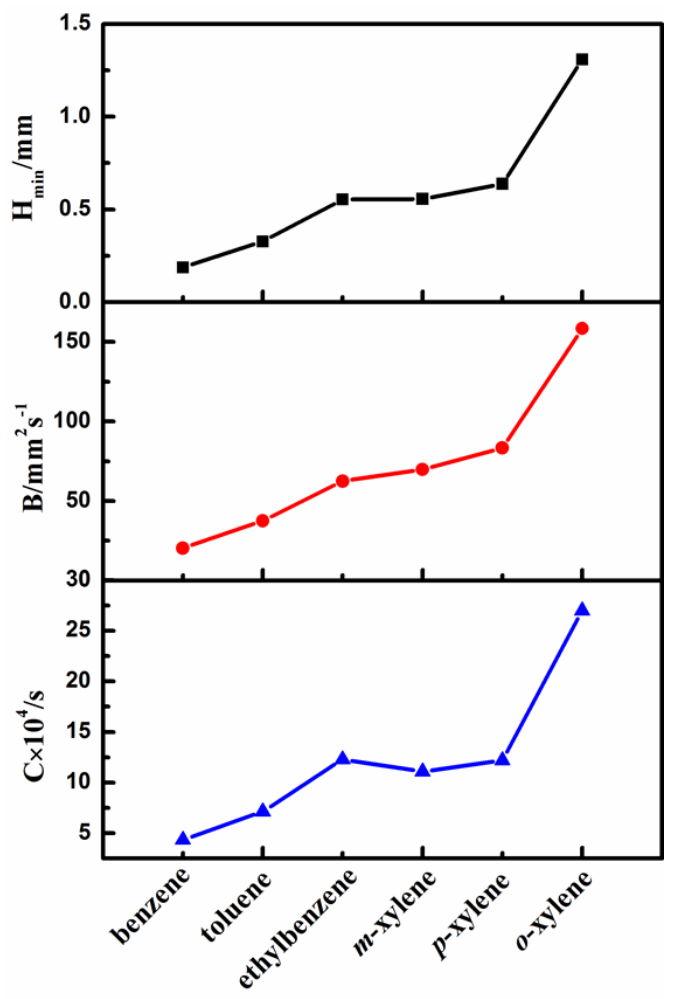


Fig. 7. Reproducible chromatograms on the KAPs-1 coated capillary column (30 m long $\times 0.32 \mathrm{~mm}$ i.d.) for separation of (a) chlorobenzenes: $180{ }^{\circ} \mathrm{C}$ for $2 \mathrm{~min}$ and then $30{ }^{\circ} \mathrm{C} \min ^{-1}$ to $250{ }^{\circ} \mathrm{C}$ (held for $2.5 \mathrm{~min}$ ) under a $\mathrm{N}_{2}$ flow rate of $0.8 \mathrm{~mL} \mathrm{~min}^{-1}$ and (b) nitrobenzenes: $210{ }^{\circ} \mathrm{C}$ for $2 \mathrm{~min}$ and then $30{ }^{\circ} \mathrm{C} \min ^{-1}$ to $250{ }^{\circ} \mathrm{C}$ (held for $2.5 \mathrm{~min}$ )

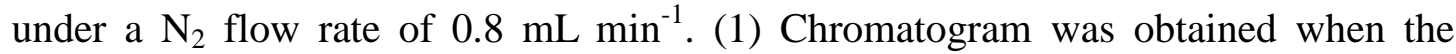
targets were first separated on the KAPs-1 coated column. $(2,3)$ Chromatograms were obtained after more than 700 injections (in the range of $50-250{ }^{\circ} \mathrm{C}$ ) and set aside for five months at the ambient conditions.
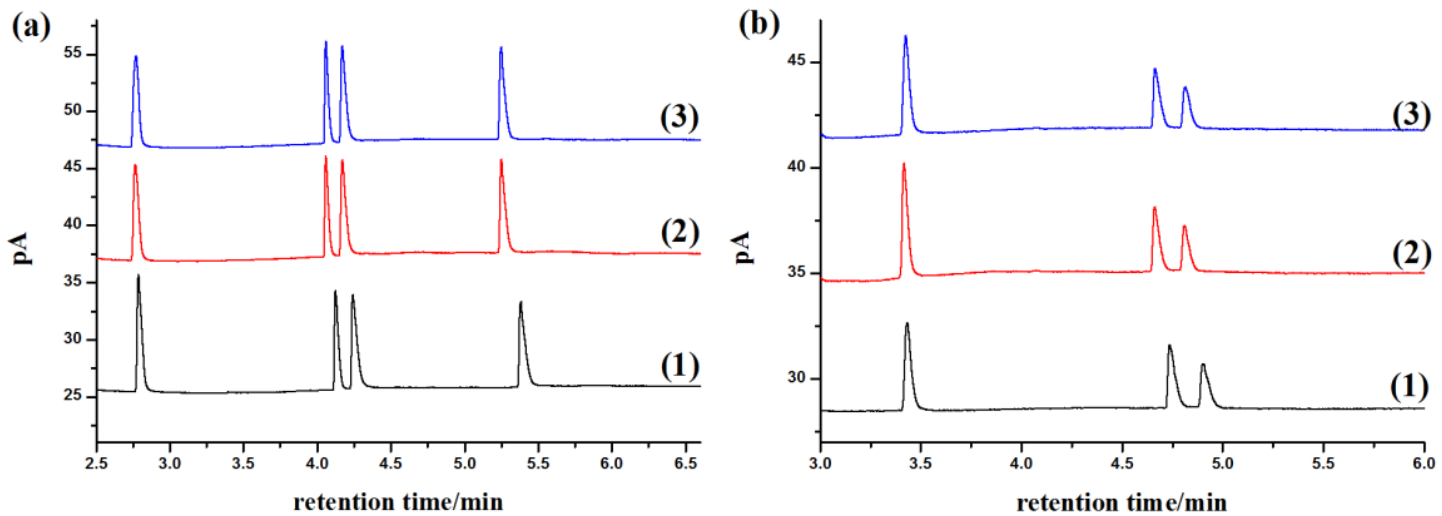
Table 1. Values of $\Delta H, \Delta S$ and $\Delta G$ for BTEXs on the KAPs-1 coated capillary column and the corresponding linearly dependent coefficients of the van't Hoff plots.

\begin{tabular}{|c|c|c|c|c|}
\hline BTEX & $\Delta H / \mathbf{k J} \cdot \mathrm{mol}^{-1}$ & $\Delta S / \mathbf{J} \cdot \mathbf{m o l}^{-1} \cdot \mathbf{K}^{-1}$ & $\Delta G / \mathbf{k J} \cdot \mathrm{mol}^{-1}$ & Adj. $R^{2}$ \\
\hline benzene & -58.2 & -107.0 & -7.6 & 0.9999 \\
\hline toluene & -67.4 & -117.5 & -11.8 & 0.9999 \\
\hline ethylbenzene & -75.6 & -127.3 & -15.3 & 0.9999 \\
\hline$m$-xylene & -76.4 & -128.5 & -15.6 & 0.9998 \\
\hline$p$-xylene & -76.8 & -128.9 & -15.8 & 0.9999 \\
\hline$o$-xylene & -76.6 & -127.7 & -16.2 & 0.9999 \\
\hline
\end{tabular}

